\title{
Synthesis and characterization of a 2D covalent organic framework (COF) of hexagonal topology using boronate linkages
}

\author{
RAMEES P P, PRADIP KUMAR MONDAL and DEEPAK CHOPRA*(1) \\ Department of Chemistry, Indian Institute of Science Education and Research Bhopal, Bhopal By-Pass Road, \\ Bhauri, Bhopal, Madhya Pradesh 462 066, India \\ E-mail: dchopra@iiserb.ac.in
}

MS received 5 February 2018; revised 17 March 2018; accepted 26 March 2018; published online 27 April 2018

\begin{abstract}
A covalent organic framework (COF) has been designed and synthesized successfully by a condensation reaction between hexahydroxytriphenylene hydrate and tetrahydroxydiborane. The characterisation studies reveal that it has good thermal stability and high crystallinity. This is a mesoporous material having a pore size of $2.8 \mathrm{~nm}$ and also having a B-B linkage with prospect of potential applications in future.
\end{abstract}

Keywords. Covalent organic framework; porosity; mesoporous; crystallinity.

\section{Introduction}

The field of porous covalent-organic frameworks $(\mathrm{COFs})^{1}$ has been developing rapidly due to their multichemical functionality. The salient features that make COFs one of the most attractive field now are predetermined and periodic diverse structures, ${ }^{2}$ permanent high porosities with low density and high surface area, ${ }^{3}$ high chemical and thermal stabilities, ${ }^{4}$ and semiconducting properties. ${ }^{5}$ All these features enable COFs to play a major role in various applications like gas adsorption, optoelectronics, catalysis, gas separation, proton conductivity, chemical sensing, drug delivery, energy storage and chromatographic separations. ${ }^{6}$ 2D COFs constructed from $\pi$-conjugated systems with short interlayer distances could exhibit electronic interactions between different sheets, and hence potentially become conducting materials. ${ }^{7}$ The intrinsic cavities in COFs bear unique advantage in selective binding and separation. ${ }^{8}$ These are the materials which mainly consist of light elements (B, C, N, O, and H). ${ }^{9}$ The concepts behind COFs are the design and synthesis of $2 \mathrm{D}$ or $3 \mathrm{D}$ crystalline organic extended porous structures in which the building blocks are linked by covalent bonds. Here, the various organic units are precisely integrated to create predesigned skeletons. ${ }^{10}$ Several linkages in

\footnotetext{
*For correspondence
}

organic reactions have been used successfully in synthesizing COFs, namely imine, boronate ester, borazine, hydrazine, boroxine, borosilicate, triazine, squaraine, and azodioxide. ${ }^{11}$ The chemical and physical properties of COFs can be easily tuned through a selection of building blocks and linkage motifs. The porous COFs can be classified into two sub-classes: crystalline and amorphous porous materials. ${ }^{12}$ The crystalline porous materials are prepared by dynamic covalent controlled reactions, where a rapid equilibrium allows the coexistence of a variety of different species among which molecules can be selected with desired properties. So, it possesses reactions with reversible characteristics having the property of self-assembly, error correction and editing. But preparation of amorphous porous materials is done by kinetically-controlled covalent reactions, which form covalent bonds irreversibly. Because of this, unlike amorphous materials, crystalline porous materials form ordered structures.

Since Yaghi and co-workers reported the first 2D COF material in $2005,{ }^{13}$ COFs have attracted increasing worldwide attention in several areas. In order to obtain predetermined COFs using building blocks and linkage motifs, the synthetic procedure plays an extremely vital role. Since COFs are prepared by dynamically-controlled covalent reactions, regulating the conditions of thermodynamic equilibrium during covalent bond formation is extremely essential in

Electronic supplementary material: The online version of this article (https://doi.org/10.1007/s12039-018-1456-x) contains supplementary material, which is available to authorized users. 


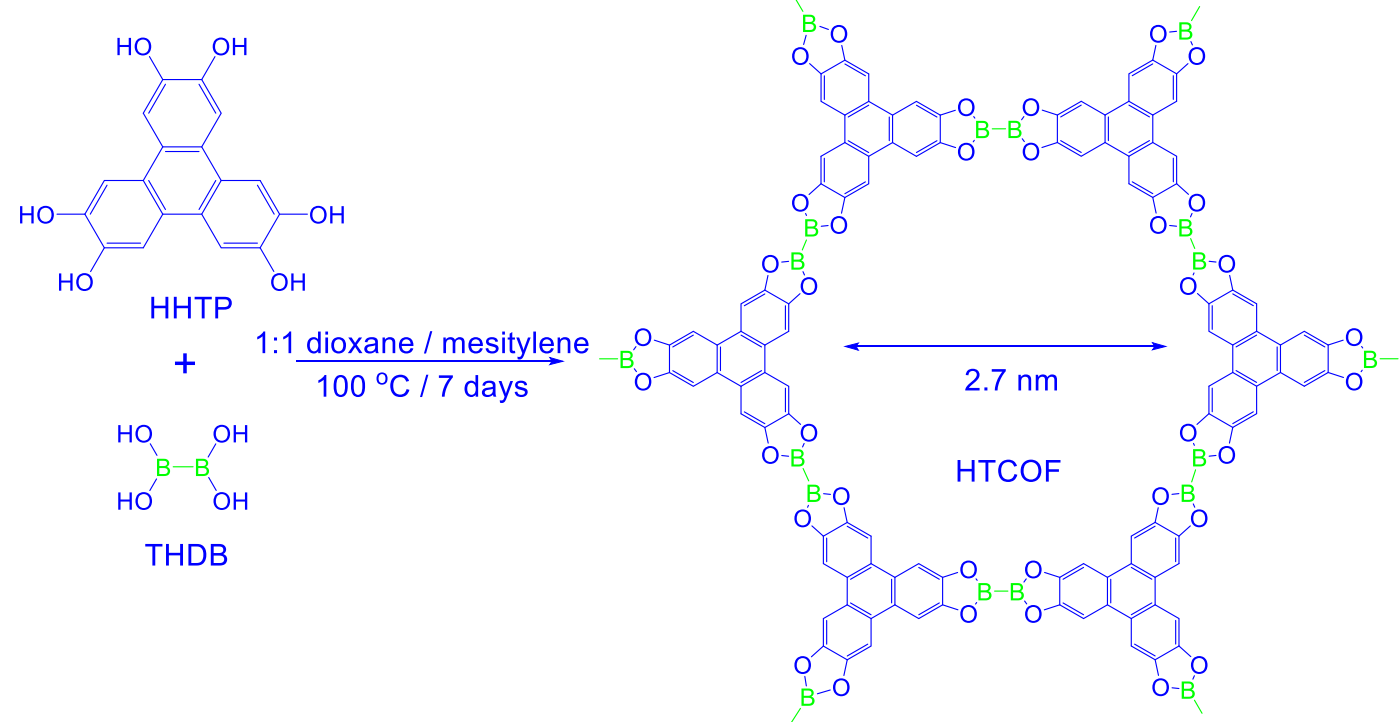

Scheme 1. The synthetic reaction of hexahydroxytriphenylene hydrate (HHTP) and tetrahydroxydiborane (THDB) to form HTCOF with a hexagonal mesoporouspore.

forming highly ordered covalent frameworks. Especially the reaction media (including solvent mixture) and conditions (temperature, pressure, and presence or absence of template) are significant. Yaghi and coworkers exploited solvothermal methods to get success in the synthesis of the first COF. ${ }^{13}$ Since then, different new types of synthetic procedures have been reported by various research groups. Amongst these, solvothermal, ionothermal, and microwave methods are most widely applied synthetic procedures for COF synthesis. Research is under progress to achieve room-temperature synthesis for COFs. ${ }^{14}$ Most of the COFs reported till now are synthesized by solvothermal method.

Herein, we report the synthesis of $2 \mathrm{D}$ hexagonal mesoporous COF (HTCOF) by employing a mixture of $\mathrm{C}_{3}$-symmetric 2,3,6,7,10,11-hexahydroxytriphenylene hydrate (HHTP) and $\mathrm{C}_{2}$-symmetric tetrahydroxydiboron (THDB) monomers in 2:3 stoichiometric ratio (Scheme 1). Both HHTP and THDB are planar molecules that display the capability to form ordered 2D porous periodic networks that are analogous to graphene material. This is the first COF ever reported having a $\mathrm{B}-\mathrm{B}$ linkage with good crystalline peaks. It forms a mesoporous material having a pore size of $2.8 \mathrm{~nm}$.

\section{Experimental}

\subsection{Synthesis of HTCOF}

The HTCOF was synthesized using solvothermal conditions by reacting 2,3,6,7,10,11-hexahydroxytriphenylene hydrate and tetrahydroxydiboron monomers in a 1:1 (v/v) 1,4 dioxane and mesitylene mixture (Scheme 1). Tetrahydroxydiboron was purchased from Sigma-Aldrich Chemicals, and 2,3,6,7,10,11-hexahydroxytriphenylene hydrate was purchased from TCI chemicals. These two starting materials were used without further purification. Schlenk tube of $25 \mathrm{~mL}$ capacity was used for COF synthesis. For washing the product, acetone was used after drying it over molecular sieves. The monomers HHTP (169.6 mg, $0.52 \mathrm{mmol})$ and THDB (70.36 mg, $0.78 \mathrm{mmol}$ ) were added to a Schlenk tube which is fitted with a nitrogen balloon. Solvents 1,4 dioxane $(5 \mathrm{~mL})$ and mesitylene $(5 \mathrm{~mL})$ were added to it using a syringe. The mixture was then sonicated for $2 \mathrm{~min}$. The reaction system was heated at $100^{\circ} \mathrm{C}$ for seven days. HTCOF was obtained in $30 \%$ yield by vacuum filtration and washed with anhydrous acetone. A green crystalline powder that was insoluble in common organic solvents was obtained.

\subsection{Powder X-ray diffraction (PXRD)}

The experimental powder X-ray diffraction pattern of the synthesised COF was recorded on PANalytical Empyrean $\mathrm{X}$-ray diffractometer with $\mathrm{CuK} \alpha$ radiation $(1.5406 \AA)$. The bulk powder of each sample was placed in a silica sample holder and measured by a continuous scan with a step size of $0.013103^{\circ}$. Pawley refinement ${ }^{15}$ was performed using Materials Studio $6.1^{16}$ on the experimental PXRD pattern.

\subsection{BET surface area and porosity measurement}

Nitrogen sorption measurements were conducted at $77 \mathrm{~K}$ to evaluate the porosity characteristics of the prepared COF. Before sorption experiments, the COF sample was degassed at $100^{\circ} \mathrm{C}$ for $24 \mathrm{~h}$. Gas adsorption experiments were performed on a QuantachromeAutosorb equipment in $9 \mathrm{~mm}$ 
w/o rod Cell. These isotherms were analyzed using ASiQwin software. Pore-size distribution profile was estimated using NLDFT method. ${ }^{17}$

\subsection{Transmission electron microscopy (TEM) imaging and Tyndall effect}

For TEM imaging and investigating the Tyndall effect, $1 \mathrm{mg}$ of HTCOF was added in $1 \mathrm{~mL}$ of acetone and sonicated for $15 \mathrm{~min}$. The sample was coated on the carbon-coated copper grid and dried at room temperature intended for TEM imaging. High-resolution TEM images were obtained in FEI Talos F200S TEM system with an accelerated voltage of $200 \mathrm{kV}$.

\section{Results and Discussion}

Even though many diboronic acid derivatives have been used in synthesizing COFs, tetrahydroxydiborane has been employed by us for the first time as a building block in COF synthesis. So, it was challenging to determine the appropriate conditions required for synthesizing the crystalline product. We have optimized the synthetic procedures by performing the reaction under different solvent conditions, apparatus, and methods. After trying in various solvent combinations and their ratios (Table S1 and Figure S1 in Supplementary Information), we obtained significantly improved yields and enhanced crystallinity in dioxane: mesitylene $(1: 1 \mathrm{v} / \mathrm{v})$ solvent combination. A series of experiments was carried out before optimizing the final conditions of the performed reaction, i.e., the duration of heating and the temperature (Table S1 and Figure S1). Finally, we obtained the synthetic method which gives the desired COF material with higher thermal stability and decent crystallinity.

Thermogravimetric analyses (TGA) were carried out on a Perkin-Elmer TGA 4000 instrument under $\mathrm{N}_{2}$ atmosphere at a heating rate of $10^{\circ} \mathrm{C} / \mathrm{min}$ with a temperature range of $30-900^{\circ} \mathrm{C}$ (Figure 1). HTCOF displayed good thermal stability, as revealed by TGA analysis, which indicates that it maintained about $85 \%$ of its weight up to $300^{\circ} \mathrm{C}$ and $70 \%$ up to $550{ }^{\circ} \mathrm{C}$.

The powder X-ray diffraction (PXRD) analysis of the obtained product confirmed the crystallinity of the prepared COF. It showed intense peaks at 2-theta values of $7.9^{\circ}, 15.4^{\circ}, 16.4^{\circ}, 20.7^{\circ}, 26.8^{\circ}, 28.1^{\circ}$, and $31.9^{\circ}$ along with some other peaks of lower diffraction intensities (Figure 2), indicating long-range ordering. The powder pattern was indexed in the hexagonal crystal system with lattice parameters $\mathrm{a}=23.03 \AA$, b $=23.03 \AA$, $\mathrm{c}=$ $5.61 \AA, \alpha=90^{\circ}, \beta=90^{\circ}$, and $\gamma=120^{\circ}$ with $\mathrm{Rp}=$ $2.76 \%$ and Rwp $=3.74 \%$ (Figure 2). In order to confirm that the phases observed from PXRD indeed corroborate with the target, we modelled the possible extended

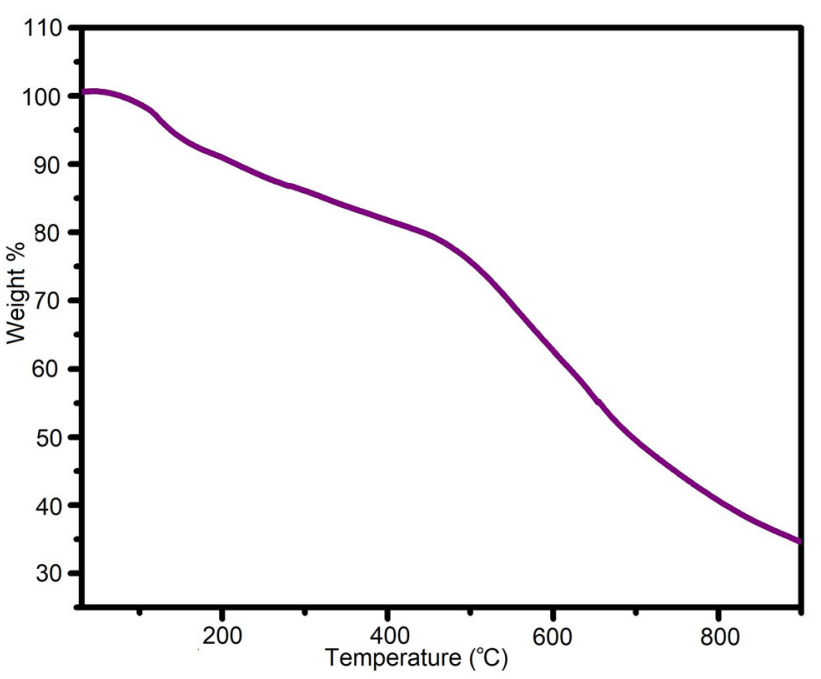

Figure 1. TGA plot of HTCOF@ $10{ }^{\circ} \mathrm{Cmin}^{-1}$.

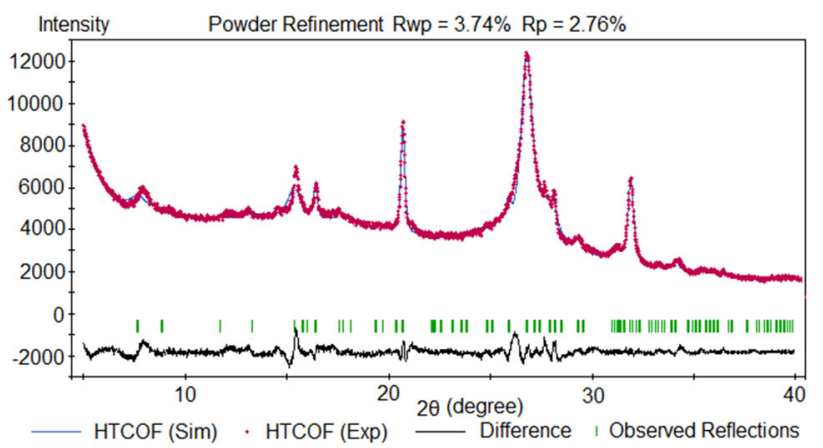

Figure 2. Experimentally observed PXRD pattern (red), Pawley refined pattern (blue), their difference (black), and observed reflections (green line) of HTCOF.

structure that could be formed from the monomers using the obtained lattice parameters. To clarify the stacked configuration of the $2 \mathrm{D}$ sheets, two extreme possible stacking models, namely, eclipsed packing and staggered packing were modelled with $P 1$ space group. ${ }^{18}$ Given that planar 2D organic sheets were expected to form, layered crystal structures were modelled, in which a possibility of eclipsed stacking, where atoms of adjacent sheets lie directly over each other (Figure 3a) and in staggered arrangement three-connected vertices lie over the centre of the six-membered rings (Figure 3b). The obtained simulated PXRD pattern of the synthesized COF is shown in Figure 3(c and d) and compared with the experimental pattern. The simulated PXRD pattern shows good agreement with the experimental powder $\mathrm{X}$-ray diffraction pattern. The higher angle simulated PXRD peaks $\left(2 \theta=10^{\circ}\right.$ to $\left.30^{\circ}\right)$ of the staggered model are relatively more intense (Figure $3 \mathrm{~d}$ ) in comparison to the simulated PXRD peaks at higher angle for the eclipsed model (Figure 3c), which was in good agreement with the experimental PXRD patterns. ${ }^{19}$ 


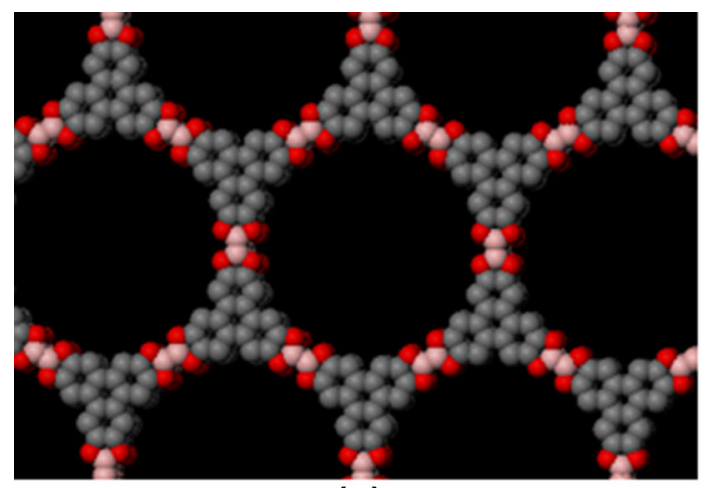

(a)

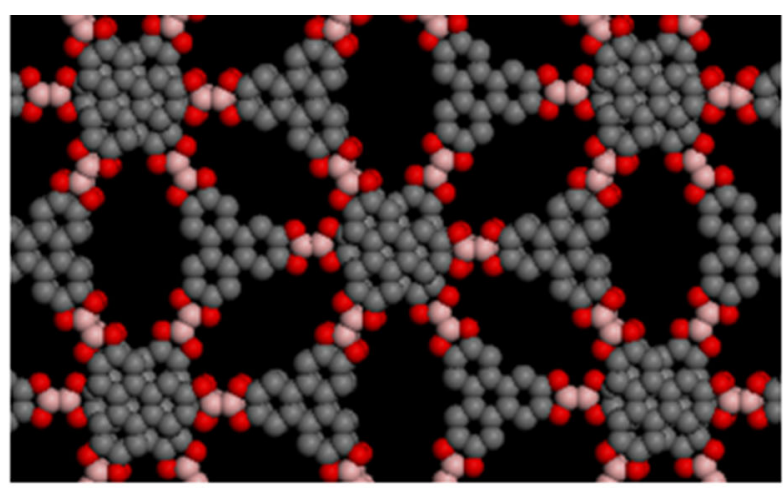

(b)

Intensity

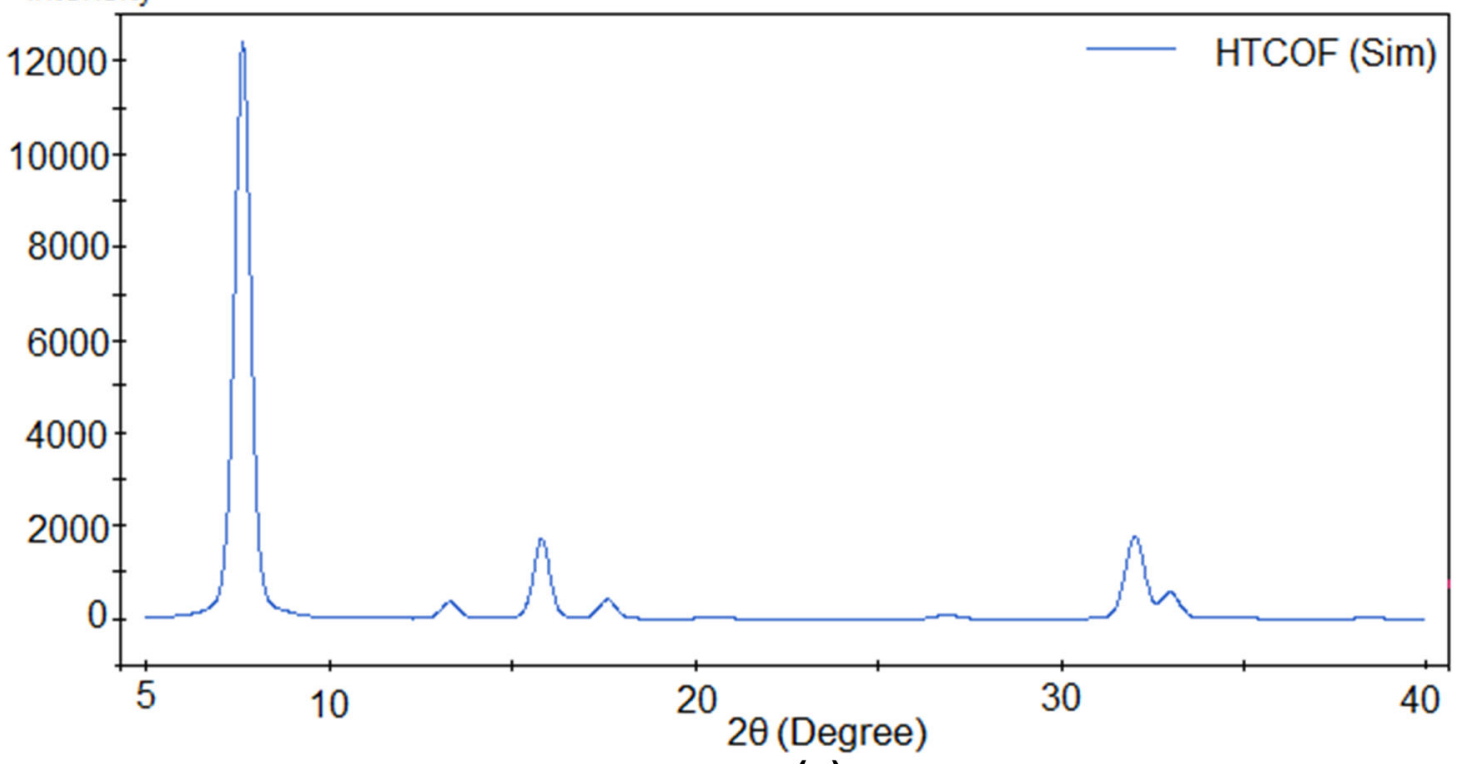

(c)

Intensity

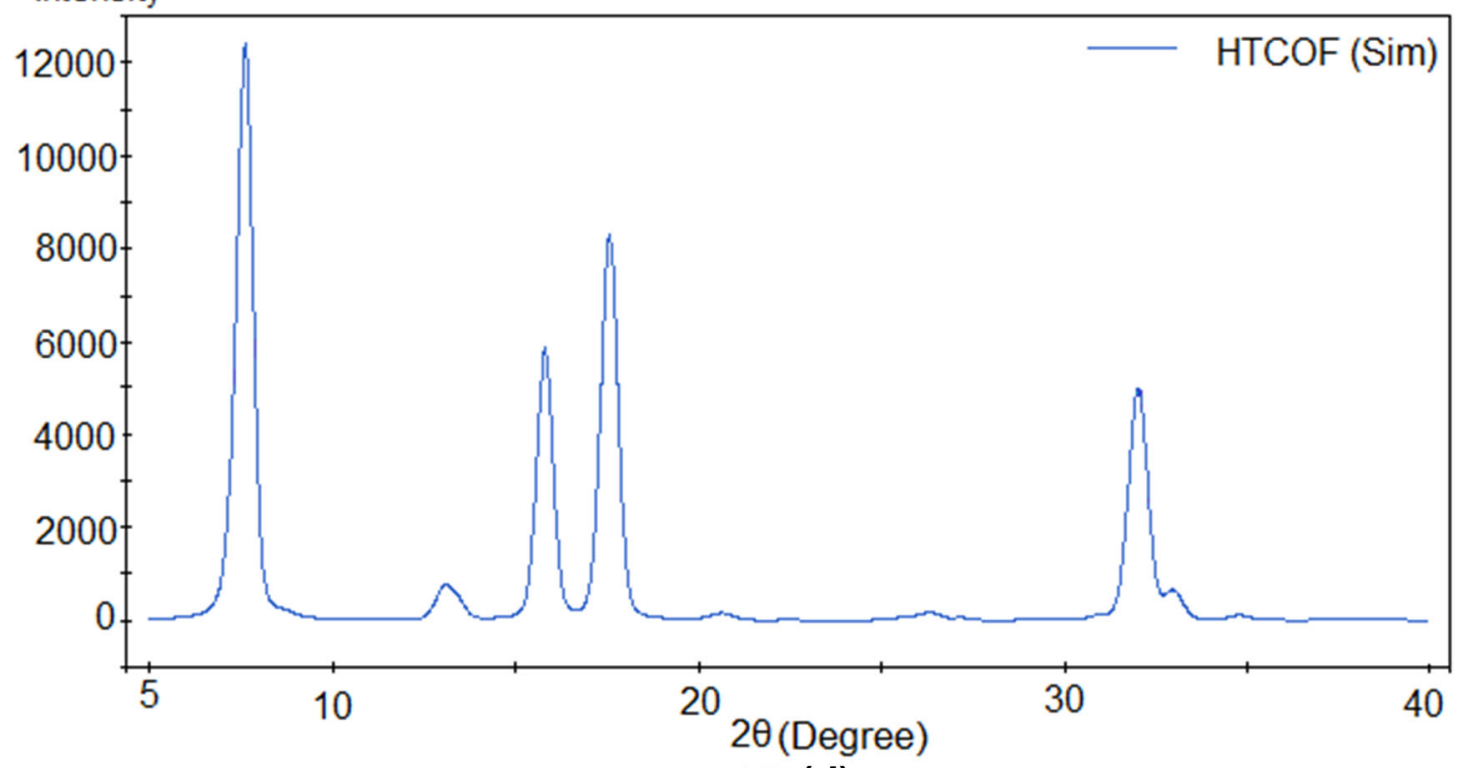

(d)

Figure 3. (a) Eclipsed packing of HTCOF; (b) Staggered packing of HTCOF; (c) Simulated PXRD pattern of eclipsed HTCOF packing; and (d) Simulated PXRD pattern of staggered HTCOF packing. 
(a)

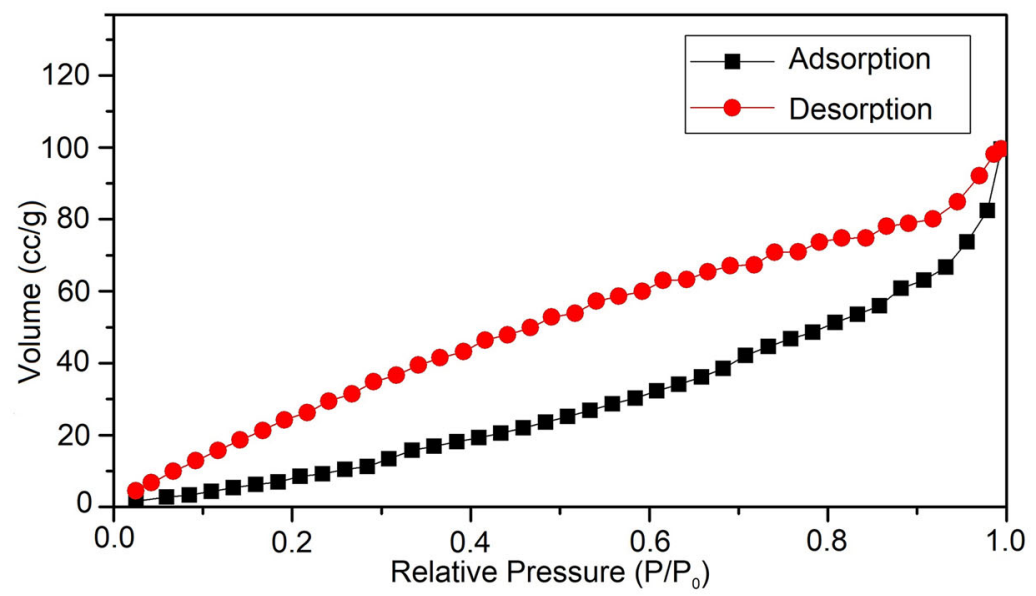

(b)
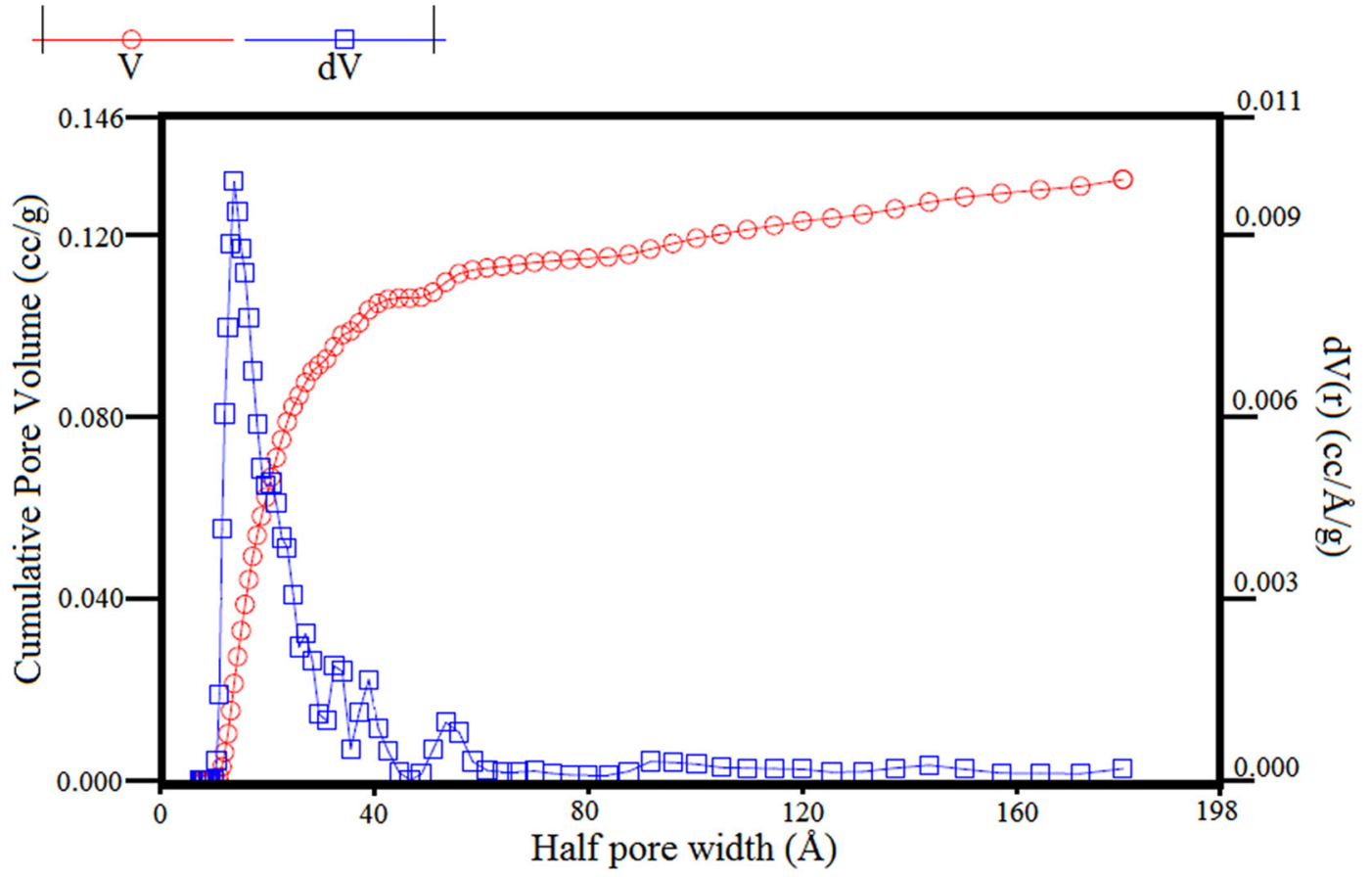

Figure 4. (a) $\mathrm{N}_{2}$ adsorption isotherm of HTCOF (black squares: adsorption; red circles: desorption). (b) Pore-size distribution profile estimated using NLDFT method (blue squares: differential pore volume; red circles: cumulative pore volume).

The $\mathrm{N}_{2}$ gas adsorption measurement confirmed the porosity of HTCOF. Figure 4a shows the nitrogen sorption isotherm. The pore size distribution was evaluated using nonlocal density functional theory (Figure $4 \mathrm{~b}$ ), and the pore size was calculated to be $2.8 \mathrm{~nm}$. This value is reliable with the theoretical pore size (2.7 $\mathrm{nm})$ predicted from the modelling based on the crystal packing. The sorption curve was classified as type II isotherm, which indicates the formation of a monolayer. The Brunauer-Emmett-Teller (BET) surface area and total pore volume were estimated to be $60 \mathrm{~m}^{2} / \mathrm{g}$ and $0.132 \mathrm{cc} / \mathrm{g}$, respectively. The hysteresis loop was observed in the whole range of HTCOF adsorption- desorption isotherm which is reliable with the high pore size (mesoporous). We attribute the lower surface area to the presence of unreacted monomers and small branched polymers trapped within the material and interpenetration of the hexagonal network. The lower total pore volume of HTCOF is reliable with the possible staggered model. Table 1 shows the comparison between some of the reported boronate esters COFs and HT-COF in terms of pore size, surface area, and volume.

Transmission electron microscopy (TEM) images was used to investigate the morphology of the HTCOF after evaporating an acetone suspension $(\mathrm{c}=1.0 \mathrm{~g} / \mathrm{L})$ on a carbon-coated copper grid. The TEM images 


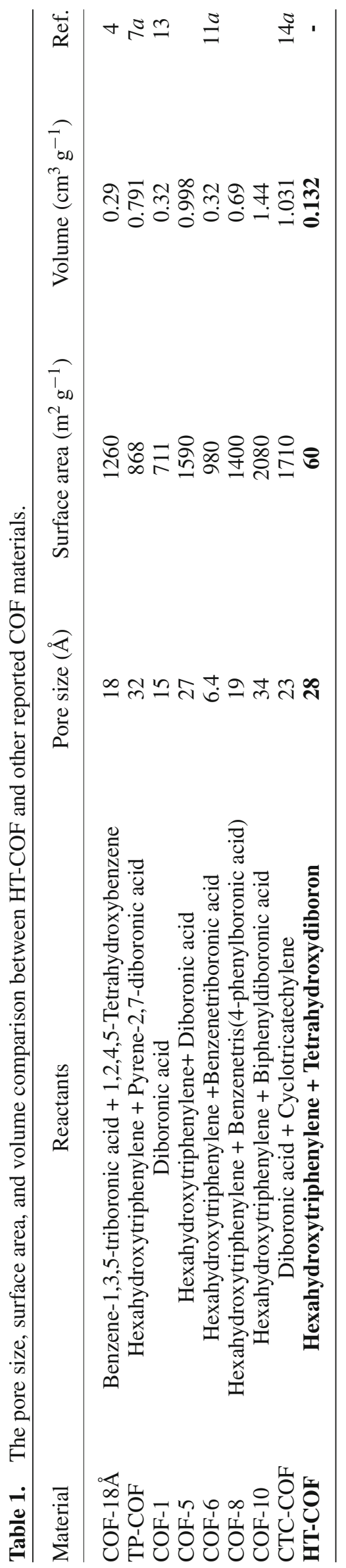

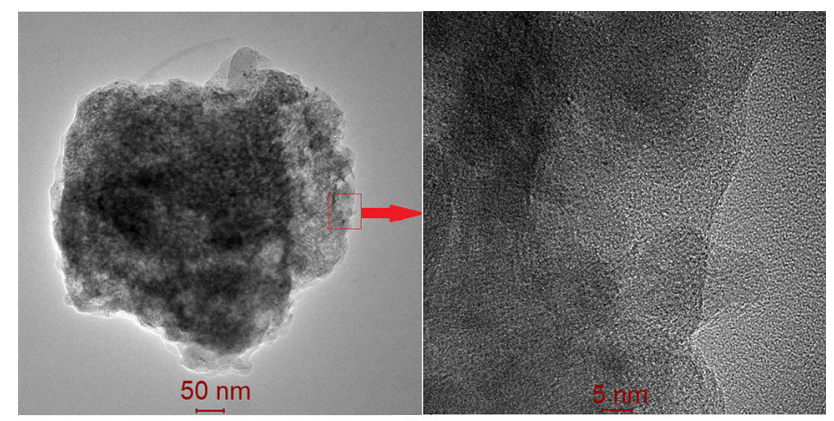

(a)

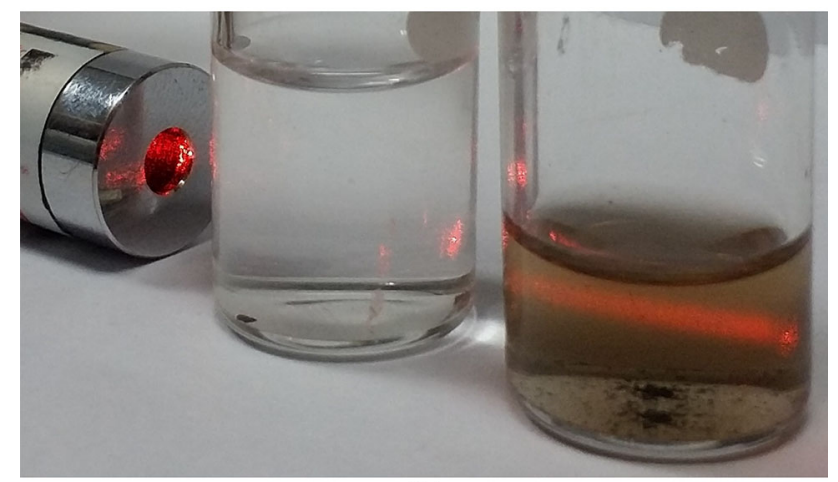

(b)

Figure 5. (a) TEM images of bulk HTCOF. (b) The Tyndall effect of a diluted HTCOF suspension using a laser pointer.

specified the presence of mesoscopic substances (Figure 5a). Upon introduction of a laser beam, the Tyndall scattering effect was observed for the diluted acetone suspension (Figure 5b).

\section{Conclusions}

In summary, we have synthesized a new 2D COF constructed by a reversible condensation reaction between hexahydroxytriphenylene hydrate and tetrahydroxydiborane. The COF obtained is highly crystalline with a pore size of $2.8 \mathrm{~nm}$ and also having a B-B linkage. Studies in future are directed towards increasing its surface area and porosity facilitating the exploration of enhanced gas uptake and storage.

\section{Supplementary Information (SI)}

Optimization of the reaction conditions and experimentally observed PXRD pattern for preparing the HTCOF are included. Supplementary Information is available at www. ias.ac.in/chemsci.

\section{Acknowledgements}

P.K.M. thanks CSIR for the senior research fellowship. We thank IISER Bhopal for research facilities (TGA, Material studio, PXRD, and BET) and infrastructure. We thank Dr A. Srivastava for help with experiments on TEM and we 
acknowledge the FIST supported TEM facility at the Department (of Chemistry) of IISER Bhopal.

\section{References}

1. (a) Biswal B P, Chandra S, Kandambeth S, Lukose B, Heine T and Banerjee R 2013 Mechanochemical Synthesis of Chemically Stable Isoreticular Covalent Organic Frameworks J. Am. Chem. Soc. 135 5328; (b) Huang N, Ding X, Kim J, Ihee H and Jiang D 2015 A Photoresponsive Smart Covalent Organic Framework Angew. Chem. Int. Edit. 54 8704; (c) Thomas A 2010 Functional Materials: From Hard to Soft Porous Frameworks Angew. Chem. Int. Edit. 498328

2. Yang H, Du Y, Wan S, Trahan G D, Jin Y and Zhang W 2015 Mesoporous 2D covalent organic frameworks based on shape-persistent arylene-ethynylene macrocycles Chem. Sci. 64049

3. (a) El-Kaderi H M, Hunt J R, Mendoza-Cortes J L, Cote A P, Taylor R E, O'Keeffe M and Yaghi O M 2007 Designed Synthesis of 3D Covalent Organic Frameworks Science 316 268; (b) Chandra S, Kandambeth S, Biswal B P, Lukose B, Kunjir S M, Chaudhary M, Babarao R, Heine T and Banerjee R 2013 Chemically Stable Multilayered Covalent Organic Nanosheets from Covalent Organic Frameworks via Mechanical Delamination $J$. Am. Chem. Soc. 13517853

4. Tilford R W, Gemmil W R, ZurLoye H C and Lavigne J J 2006 Facile Synthesis of a Highly Crystalline, Covalently Linked Porous Boronate Network Chem. Mater. 185296

5. Dogru M and Bein T 2014 On the road towards electroactive covalent organic frameworks Chem. Commun. 505531

6. (a) Zeng Y, Zou R and Zhao Y 2016 Covalent Organic Frameworks for $\mathrm{CO}_{2}$ Capture Adv. Mater. 28 2855; (b) Nagai A, Chen X, Feng X, Ding X, Guo Z and Jiang D 2013 A Squaraine-Linked Mesoporous Covalent Organic Framework Angew. Chem. Int. Edit. 52 3770; (c) Berlanga I, Ruiz-González M L, González-Calbet J M, Fierro J L G, Mas-Ballesté R and Zamora F 2011 Delamination of Layered Covalent Organic Frameworks Small 71207

7. (a) Wan S, Guo J, Kim J, Ihee H and Jiang D 2008 A Belt-Shaped, Blue Luminescent, and Semiconducting Covalent Organic Framework Angew. Chem. Int. Edit. 47 8826; (b) Miro P, Audiffred M and Heine T 2014 An atlas of two-dimensional materials Chem. Soc. Rev. 43 6537

8. Ma H, Ren $\mathrm{H}$, Meng $\mathrm{S}$, Yan Z, Zhao H, Sun F and Zhu G 2013 A 3D microporous covalent organic framework with exceedingly high $\mathrm{C}_{3} \mathrm{H}_{8} / \mathrm{CH}_{4}$ and $\mathrm{C}_{2}$ hydrocarbon/ $\mathrm{CH}_{4}$ selectivity Chem. Commun. 499773
9. Xiang Z and Cao D 2013 Porous covalent-organic materials: synthesis, clean energy application and design $J$. Mater. Chem. A 12691

10. Feng X, Ding X and Jiang D 2012 Covalent organic frameworks Chem. Soc. Rev. 416010

11. (a) Cote A P, El-Kaderi H M, Farukawa H, Hunt J R and Yaghi O M 2007 Reticular Synthesis of Microporous and Mesoporous 2D Covalent Organic Frameworks $J$. Am. Chem. Soc. 129 12914; (b) Baldwin L A, Crowe J W, Shannon M D, Jaroniec C P and Mcgrier P L 2015 2D Covalent Organic Frameworks with Alternating Triangular and Hexagonal Pores Chem. Mater. 27 6169; (c) Dalapati S, Jin E, Addicoat M, Heine T and Jiang D 2016 Highly Emissive Covalent Organic Frameworks J. Am. Chem. Soc. 138 5797; (d) Xu S Q, Zhan T G, Wen Q, Pang Z F and Zhao X 2016 Diversity of Covalent Organic Frameworks (COFs): A 2D COF Containing Two Kinds of Triangular Micropores of Different Sizes ACS Macro Lett. 5 99; (e) Uribe-Romo F J, Doonan C J, Furukawa H, Oisaki K and Yaghi O M 2011 Crystalline Covalent Organic Frameworks with Hydrazone Linkages J. Am. Chem. Soc.133 11478

12. Lanni L M, Tilford R W, Bharathy M and Lavigne $\mathbf{J} \mathbf{J}$ 2011 Enhanced Hydrolytic Stability of Self-Assembling Alkylated Two-Dimensional Covalent Organic Frameworks J. Am. Chem. Soc. 13313975

13. Cote A P, Benin A I, Ockwig N W, O'Keeffe M and Matzger A J and Yaghi O M 2005 Porous, Crystalline, Covalent Organic Frameworks Science 3101166

14. (a) Yu J T, Chen Z, Sun J, Huang Z T and Zheng Q Y 2012 Cyclotricatechylene based porous crystalline material: Synthesis and applications in gas storage J. Mater. Chem. 22 5369; (b) Ding S Y and Wang W 2013 Covalent organic frameworks (COFs): from design to applications Chem. Soc. Rev. 42548

15. Engel G E, Wilke S, Konig O, Harris K D M and Leusen F J J 1999 PowderSolve- a complete package for crystal structure solution from powder diffraction patterns $J$. Appl. Cryst. 321169

16. Accelrys. 2008 Material Studio Release Notes Release 4.4; Accelrys Software: San Diego

17. Jagiello J and Olivier J P 2013 2D-NLDFT adsorption models for carbon slit-shaped pores with surface energetical heterogeneity and geometrical corrugation Carbon $\mathbf{5 5} 70$

18. Guo J, Xu Y, Jin S, Chen L, Kaji T, Honsho Y, Addicoat M A, Kim J, Saeki A, Ihee H, Seki S, Irle S, Hiramoto M, Gao J and Jiang D 2013 Conjugated organic framework with three-dimensionally ordered stable structure and delocalized $\pi$ clouds Nat. Commun. 4. 2736 https:// doi.org/10.1038/ncomms3736

19. Lukose B, Kuc A and Heine T 2011 The Structure of Layered Covalent-Organic Frameworks Chem. Eur. J. 172388 